\title{
Factores que intervienen en el manejo del dolor oncológico: un reto para el profesional de enfermería
} Factors involved in the management of cancer pain: a challenge for nursing professionals

\section{Causas que intervém nos cuidados da dor em pacientes de oncologia: retos para os profissionais de enfermagem}

\author{
Wilmer Orlando Chávez-Cañas, Enf., Esp. * \\ Claudia Patricia Becerra-Cristancho, Enf. ** \\ Angie Sídney Naranjo-García, Enf. ***
}

\section{Resumen}

Introducción: El dolor es el síntoma principal en la mayoría de los pacientes que padecen de cáncer. La evidencia epidemiológica demuestra que en todo el mundo existen 37.1 millones de casos de cáncer al año, los cuales ocasionan 6.9 millones de muertes. Por desgracia, muchos de los pacientes que durante algún estadio de su enfermedad sufren dolor, reciben poca o ninguna atención. Informes de Twycross, tras estudio de 2,000 pacientes, sugieren que la mayoría de ellos, no reciben un alivio satisfactorio del dolor. Objetivo: Identificar en la literatura reportada en las bases de datos los factores que intervienen en el manejo del dolor del paciente oncológico y el rol de enfermería en este manejo. Metodología: Se hizo revisión sistematizada de la literatura entre los años 2001 y 2014 utilizando los descriptores MeSH: "pain management", "oncology" and "nursing", en las bases de datos Pubmed, Ebsco, ProQuest, Scielo, y Ovid. Resultados: la literatura reporta que son múltiples los factores que intervienen en el manejo del dolor por parte de los profesionales de enfermería tales como la poca credibilidad que el profesional de enfermería tiene, para aceptar como cierta la intensidad del dolor que el paciente refiere, así como la inadecuada evaluación de la intensidad del dolor, entre otros. Conclusiones: Se requiere que el profesional de enfermería posea conocimientos sobre el manejo del dolor oncológico, ya que es parte del equipo médico y es quien asiste permanentemente al paciente, lo que le permite determinar la eficacia del tratamiento y comentar la evolución para hacer los respectivos cambios. [Chávez-Cañas WO, BecerraCristancho CB, Naranjo-García AS. Factores que intervienen en el manejo del dolor oncológico: un reto para el profesional de enfermería. MedUNAB 2016; 19(1): 18-24]

Palabras clave: Enfermería Oncológica; Manejo del Dolor; Rol de la Enfermera; Personal de Enfermería; Atención de Enfermería; Procesos de Enfermería.

\begin{abstract}
Introduction: Pain is one of the most feared symptoms of cancer patients, representing the main symptom in most patients who suffer from it. Epidemiological evidence shows that worldwide there are 37.1 million cases of cancer annually,
\end{abstract}

\footnotetext{
* Enfermero, Especialista en Enfermería Oncológica. Clínica Carlos Ardila Lülle. Instituto Nacional de Cancerología, Colombia.

** Estudiante de Enfermería Práctica Electiva de Profundización. Fundación Cardioinfantil. Bogotá, Colombia, Universidad Autónoma de Bucaramanga, Colombia.

***Estudiante VII semestre de Enfermería, Universidad Autónoma de Bucaramanga, Colombia.
}

Autor de correspondencia: Wilmer Orlando Chávez-Cañas, dirección de correspondencia: calle 153 № 21-15 Apartamento 301, Floridablanca, Santander, Colombia. Correo electrónico: hematoonco7@gmail.com. 
which cause 6.9 million deaths. Unfortunately, many of the patients, who during some stage of their illness suffer pain, receive little or no medical attention. Twycross reports, after the study of 2,000 patients, suggest that most of them do not receive a satisfactory pain relief. Objective: To identify, in the reviewed literature in databases, the factors involved in pain management of cancer patients and the role of nursing in its handling. Methodology: A systematic review of the literature between 2001 and 2014 was performed using the MeSH descriptors: "pain management", "oncology" and "nursing" in PubMed, EBSCO, ProQuest, Scielo, and Ovid databases. Results: the literature reports that there are multiple factors involved in pain management by nursing professionals, such as low credibility that the nurse has when accepting, as true, the intensity of pain referred by the patient, as well as the improper evaluation of pain intensity among others. Conclusion: It is required that the nurse has the knowledge about the management of cancer pain because he/she is part of the medical staff and who permanently assists the patient, which allows to determine the effectiveness of the treatment and discuss the evolution to do the corresponding changes. [Chávez-Cañas WO, Becerra-Cristancho CB, NaranjoGarcía AS. Factors involved in the management of cancer pain: a challenge for nursing professionals. MedUNAB 2016; 19(1): 18-24]

Key words: Oncology Nursing; Pain Management; Nurse's role; Nursing Staff; Nursing Care; Nursing Process.

\section{Resumo}

Introdução: A dor é o principal sintoma, na maioria dos pacientes que padecem de câncer, sendo este o que mais

\section{Introducción}

El cáncer es una patología que genera un impacto no solo en la comunidad del área de la salud, sino en la comunidad en general por los efectos que causa en las personas junto con las altas cifras de mortalidad, a pesar de las múltiples investigaciones y avances que se hacen al respecto, siendo una de las principales causas de muerte en el mundo, tanto en países desarrollados como en vía de desarrollo, siendo los más frecuentes; el cáncer de pulmón, de hígado, de estómago, de colon y de mama (1). A nivel mundial 32 millones de personas padecen cáncer. En el mundo, en el 2012 causó la muerte de más de 8.2 millones de personas y se prevé que los casos anuales de cáncer aumentarán de 14 millones en el 2012 a 22 millones en las próximas dos décadas (2, 3). El proyecto GLOBACAN (2008-2012) menciona la incidencia, prevalencia y mortalidad a nivel mundial señalando que en regiones más desarrolladas el cáncer con mayor incidencia es el de seno y en las menos desarrolladas la alta incidencia es para el cáncer de pulmón, con la mayor tasa de mortalidad tanto para países desarrollados y menos desarrollados el cáncer de pulmón (3). En Colombia se registraron más de 32,000 defunciones por cáncer en el año 2009 y es la tercera causa de muerte después de las enfermedades cardiovasculares y homicidios eles temem. A evidência epidemiológica mostra que no mundo, existem 37.1 milhões de casos de câncer por ano, causando 6.9 milhões de mortes. Infelizmente, muitos dos pacientes durante algum estágio de sua doença sofrem de dor e recebem pouca ou nenhuma atenção. Twycross, após um estudo com 2.000 pacientes relata que a maioria deles não receberam alívio satisfatório da dor. Objetivo: Identificar na base de dados da literatura consultada os fatores implicados no tratamento da dor de pacientes com câncer e o rol de profissionais da enfermagem nestes casos. Metodologia: Se fez uma revisão sistemática da literatura entre 2001 e 2014 utilizando os descritores MeSH: "pain management", "oncology" and "nursing", na base de dados Pubmed, Ebsco, ProQuest, Scielo e Ovid. Resultados: A literatura relata que há vários fatores que intervém no tratamento da dor, tais como: pouca credibilidade do profesional da enfermagem em aceitar a dor forte que expresa o paciente, igualmente existe uma avaliação inadequada da intensidade da dor, etc. Conclusão: É necessária que os profissionais da enfermagem saibam como intervir na dor com pacientes de oncologia porque eles fazem parte da equipe médica e estão sempre, atendendo os pacientes. Este conhecimento Ihes permite avaliar o tratamento e comentar sua evolução para fazer as mudanças que se considerem necessárias. [Chávez-Cañas WO, Becerra-Cristancho CB, Naranjo-García AS. Causas que intervém nos cuidados da dor em pacientes de oncologia: retos para os profissionais de enfermagem. MedUNAB 2016; 19(1): 18-24]

Palavras Chave: Enfermagem Oncológica; Papel do Profissional de Enfermagem; Manejo da Dor; Recursos Humanos de Enfermagem; Cuidados de Enfermagem, Processos de Enfermagem.

$(4,5)$. Este tipo de tendencias es frecuente en otras ciudades del país como por ejemplo Bucaramanga Santander (6). La evidencia epidemiológica demuestra que en todo el mundo existen 37.1 millones de casos de cáncer al año, los cuales ocasionan 6.9 millones de muertes (7). Por desgracia, muchos de los pacientes que durante algún estadio de su enfermedad sufren dolor, reciben poca o ninguna atención.

Informes de Twycross, tras estudio de 2,000 pacientes, sugieren que la mayoría de ellos, no reciben un alivio satisfactorio del dolor; por eso, se considera uno de los, síntomas más temidos de los enfermos con cáncer (8). La Asociación Internacional para el Estudio del Dolor (IASP) lo define como "una experiencia sensorial y emocional desagradable, asociada a una lesión presente o potencial de los tejidos" (9). Es, por tanto, una experiencia subjetiva: su vivencia depende de las características de la persona que lo experimenta. Este aspecto debe tenerse siempre en cuenta a la hora de planificar el tratamiento, que no debe limitarse tan sólo en las medidas farmacológicas. Por tanto, deberían identificarse los componentes físicos y los no físicos que exacerban el dolor y tener presente el concepto de «dolor total» o sufrimiento, que engloba el estímulo físico con los factores psicológicos, sociales y espirituales de las personas afectadas, su contexto y su significado (10). Para ello el 
Tabla 1. Clasificación del dolor

\begin{tabular}{|c|c|c|}
\hline INTENSIDAD & DURACIÓN & CARACTERÍSTICAS \\
\hline $\begin{array}{c}\text { Escala Visual Análoga del } \\
\text { Dolor (EVA) (16) }\end{array}$ & $\begin{array}{l}\text { Agudo: } \\
<6 \text { meses, con signos y síntomas }\end{array}$ & $\begin{array}{l}\text { Visceral: } \\
\text { constante, sordo, mal localizado (20). }\end{array}$ \\
\hline Leve: 0-3 & objetivos (17). Predominantemente un & Somático: constante, intenso, bien \\
\hline Moderado: 4-6 & síntoma o manifestación de lesión & localizado (21). \\
\hline Intenso: 7-10 & tisular (18). & Neuropático: \\
\hline \multirow[t]{5}{*}{ Muy intenso: > 10} & Crónico: & es un dolor que se produce por una \\
\hline & $>6$ meses, sin signos objetivos (19). & lesión del sistema nervioso central o \\
\hline & Se considera como una enfer medad & periférico (22). \\
\hline & en sí mismo, de difícil tratamiento; y & Impredecible: sin causa aparente \\
\hline & $\begin{array}{l}\text { tiene repercusiones negativas sobre } \\
\text { calidad de vida (18) }\end{array}$ & conocida y puede durar de minutos a \\
\hline
\end{tabular}

Fuente: Construida por los autores tomando como referencia la literatura revisada

personal de la salud debe entender que el paciente debe ser tratado desde un punto de vista biopsicosocial, así como conocer una serie de manifestaciones y necesidades (11).

El dolor está presente en un 30\% de los pacientes en el momento del diagnóstico y en un $70-80 \%$ de los pacientes en las fases finales de la enfermedad (12). Teniendo en cuenta el rol central que desempeña el trabajador de enfermería en el cuidado de los pacientes que sufren dolor, se hace necesario capacitar al futuro profesional, en el conocimiento de la fisiopatología del dolor, teorías epidemiológicas del dolor, síndromes dolorosos más frecuentes, variables que pueden influenciar la percepción del paciente y su respuesta al dolor, la determinación clínica por métodos válidos y confiables, medicamentos útiles en el alivio del dolor, terapéuticas invasivas y no invasivas en el tratamiento del mismo, ciencias básicas e investigación aplicada al manejo y alivio del dolor, destrezas técnicas, psicológicas y de conocimiento que deberá aplicar el profesional de enfermería (13). También es importante contemplar los aspectos culturales que contribuyan con un adecuado manejo del dolor, es así, como cada vez más es de interés para los profesionales desarrollar la competencia cultural para integrarla en los planes de cuidado que piensen plantear con el objeto de tener mejores resultados de la salud (14).

Por otra parte, el dolor oncológico se puede clasificar de distintas maneras en función de su intensidad, duración o de sus características como se aprecia en la (Tabla 1) (15-23).

Una vez detectada su existencia, es importante etiquetarlo según las normas de clasificación que se han propuesto anteriormente, no sólo para la prescripción terapéutica, sino principalmente para su seguimiento, monitorización de la respuesta y modificaciones en el tratamiento (24).

En el momento del diagnóstico de un proceso neoplásico, entre el $30-50 \%$ de los pacientes presentan dolor; en las fases avanzadas de la enfermedad puede llegar al 70-90\% (22). El 20\% de los pacientes refieren un dolor intenso, siendo moderado en el $30 \%$ y de intensidad leve en el $24 \%$. Los tumores más dolorosos son los óseos $85 \%$, cérvix, cabeza, cuello, y estómago, siendo menos frecuentes los tumores de linfoma y leucemia 5\% (12). Para el manejo del dolor existe un equipo interdisciplinario, entre ellos el enfermero especialista en oncología cuyo rol consiste en saber diferenciar y manejar el tipo de dolor que presentan los pacientes en las distintas etapas de la enfermedad, ya que el manejo del dolor es considerado un fenómeno multidimensional que requiere una valoración completa que permita obtener datos objetivos y subjetivos sobre las respuestas fisiológicas, cognitivas y emocionales del paciente (25-27).

Es importante recordar que el dolor percibido por el paciente, afecta su calidad de vida y la del cuidador. Con frecuencia la valoración del dolor es unidimensional ya que se basa sólo en respuestas fisiológicas, olvidando que el dolor como toda experiencia emocional es subjetivo; sólo el paciente conoce el dolor y cuánto le duele, por eso su valoración debe basarse necesariamente en el informe del paciente cuando puede verbalizarlo; cuando al paciente se le dificulta verbalizar el grado de dolor, se valora de acuerdo a la teoría de síntomas desagradables, la cual hace referencia a tres componentes que son: síntomas, factores que afectan la naturaleza del síntoma y consecuencias de la experiencia del síntoma. Se ha de tener en cuenta además, cuatro dimensiones de cada síntoma que son: intensidad o gravedad, grado de estrés o ansiedad, tiempo y calidad (28).

Por otra parte, es necesario reconocer que el profesional de enfermería realiza intervenciones basadas en la evidencia científica, utilizando la metodología del proceso de atención de enfermería (PAE), junto con el lenguaje estandarizado NANDA-NOC-NIC. En esta metodología se identifican los diagnósticos principales en los pacientes oncológicos según la American Nursing Diagnosis Association (NANDA), uno de ellos es el "dolor crónico" identificado con el código (00133), el cual es definido como una experiencia sensitiva y emocional desagradable ocasionada por una lesión tisular real o potencial (29); de inicio súbito o 
lento de cualquier intensidad (leve a grave), constante o recurrente sin un final anticipado o previsible y una duración superior a seis meses. El profesional de enfermería a través de la Nursing Interventions Classification (NIC) pone en práctica uno de los múltiples cuidados para alivio del dolor que es la intervención "manejo del dolor" identificado con el código (1400) en el que define el alivio del dolor o disminución del dolor cuando se llega a un nivel de tolerancia que sea aceptable para el paciente $(30,31)$.

Es de reconocer que en la práctica clínica de enfermería se vienen haciendo esfuerzos por incluir dentro de la practica guías con evidencia científica que ayuden a unificar los criterios más apropiados para el manejo del dolor, en este sentido existen organizaciones como Registered Nurses Association of Ontario (RNAO) quienes vienen promoviendo la implementación de guías de práctica clínica para la valoración y manejo del dolor, en diferentes países, al igual que creando indicadores que permitan identificar la calidad de la atención de enfermería (32-34).

En la práctica clínica de la enfermería oncológica la valoración del paciente es indispensable incluyendo medidas como la utilización de escalas de medición, entre ellas la escala visual análoga y otras (35-37) pues permite identificar y ubicar en el contexto social, cognitivo y de salud como se encuentra el paciente respecto al dolor para poder controlar el dolor, cuya responsabilidad es esencial durante todo el proceso de la enfermedad (38). No obstante, existen falencias en cuanto al tratamiento del dolor, tano en enfermeros sin posgrado, como en aquellos con especialización en oncología, tales como una correcta evaluación, valoración y tratamiento del dolor, que incluye medidas farmacológicas y no farmacológicas, creencias y prejuicios, además de mitos relacionados con la dependencia y adicción a opioides por parte de los pacientes oncológicos que requieren ser modificadas (39-41). De esta manera, resulta relevante realizar una búsqueda sistematizada de artículos de investigación, que permita identificar los factores que intervienen en el manejo del dolor del paciente oncológico y el rol de enfermería en este manejo, para que el profesional de enfermería pueda plantearse estrategias a fin de eliminar las falencias que impiden un manejo correcto del dolor oncológico $(42,43)$.

\section{Metodología}

Se realizó búsqueda sistematizada de la literatura en las bases de datos PubMed, Sientific Library (Scielo), Ebsco, ProQuiest y Ovid. Se usaron los descriptores DeCS y MeSH "enfermería oncológica", "manejo del dolor", "rol de la enfermera", "personal de enfermería" y "atención de enfermería". Los criterios de inclusión que se tuvieron en cuenta fueron artículos publicados en el período de 2005 a 2014, escogiendo aquellos que permitieran evidenciar los factores que intervienen en el manejo del dolor del paciente oncológico y el rol de enfermería para aliviarlo. Los criterios de exclusión fueron los que no cumplían con los criterios de inclusión. Para esta revisión no se tuvo en cuenta la edad de los pacientes ni el tipo de cáncer, como tampoco el tiempo de padecimiento. Se identificaron 462 artículos de investigación de los cuales se revisaron los resúmenes para verificar su pertinencia con el objetivo propuesto. Se seleccionaron 180 resúmenes y una vez leídos los artículos de texto completos fueron seleccionados 24 que cumplían con el objetivo de esta revisión.

\section{Resultados}

De acuerdo con la información de los artículos revisados se encontró que son múltiples los factores que intervienen en el manejo del dolor por parte de los profesionales de enfermería, como, por ejemplo, la poca credibilidad que el profesional de enfermería tiene, para aceptar como cierta la intensidad del dolor que el paciente refiere (39).

También, la inadecuada evaluación de la intensidad del dolor, las enfermeras no utilizan escalas de medición del dolor de manera sistematizada y la mayoría de ellas no recomienda alguna medida paliativa para mitigar dicho síntoma (44), es una de las principales barreras para su control, al igual que el desconocimiento de los efectos secundarios de los analgésicos y el temor a las reacciones adversas de los opioides, limitando así la terapéutica del dolor $(45,46)$.

Diversos autores afirman que la falta de conocimiento del profesional de enfermería de los métodos farmacológicos y no farmacológicos, las equivalencias medicamentosas y el uso de opioides son barreras presentes al momento de intervenir el dolor $(47,48)$.

Uno de los limitantes en el manejo del dolor, es el no indagar las creencias del paciente sobre el mismo; si esto se hiciera se favorecería la autogestión, la autoeficacia y la adhesión al tratamiento del mismo $(49,50)$.

Un estudio logró identificar en el profesional de enfermería el déficit de conocimiento sobre la escala analgésica establecida por la OMS; otro dato relevante es que los profesionales no conocen sobre las intervenciones de enfermería para determinar la ubicación, características, calidad y gravedad del dolor antes de administrar el fármaco al paciente; tampoco conocen sobre la intervención de enfermería de administrar analgésicos o fármacos complementarios cuando sea necesario para potenciar la analgesia. Además, se logró evidenciar que los profesionales de enfermería no conocen sobre la intervención de enseñar el uso de analgésicos, estrategias para disminuir los efectos secundarios y expectativas de implicación en la decisión sobre el dolor, conocimiento que es necesario debido a que existe analgesia controlada por el paciente dándole a este un sentido de control y una menor ansiedad (51). 
De otra parte, según declaración de International Society of Nurses in Cancer Care (ISNCC) Pain Position Statement, las barreras para el buen control del dolor han sido categorizadas en tres áreas: falta de conocimiento de los profesionales de la salud, mitos y conceptos errados por parte de los pacientes y familias, y sistemas de salud, legislación y políticas inadecuadas (42).

Todo lo anterior destaca que el rol de enfermería en el manejo del dolor del paciente oncológico en el área asistencial se basa en 4 aspectos, los cuales deben tenerse en cuenta y ponerse en práctica para poder cumplir con:

- Valoración del paciente: el profesional de enfermería debe estar en la capacidad de hacer un correcto examen físico al paciente, de manera tal que le permita identificar las necesidades del mismo y así, saber hacia dónde dirigir su plan de cuidado. Así mismo, debe recordar que el paciente es un ser holístico, por lo que debe mirar todos los aspectos del mismo, incluyendo sus creencias religiosas, su cultura, su estado físico, su estado emocional, su estado cognitivo, la perspectiva familiar, sus mitos acerca del tratamiento farmacológico, entre otros, ya que estos influyen en el manejo del dolor (42).

- Valoración del nivel del dolor: se debe usar las escalas apropiadas para ello, que son: categoriales, numéricas o analógico-visuales. Aunque existe otro tipo de escalas, como la pictórica, la de descriptores o la gráfica también utilizada en este ámbito, estas cuentan con evidencias muy limitadas sobre sus propiedades psicométricas (10). Esta evaluación se debe hacer de manera regular y debe contemplar varios contextos, ya que el dolor en pacientes oncológicos cambia con el tiempo, tanto en localización como en su extensión, sucede a diferente velocidad, muy lento y predecible o muy rápido e impredecible, se manifiesta de manera diferente en todos los pacientes.

- Intervención en el manejo del dolor: para realizar una correcta intervención, el profesional de enfermería debe contar con un amplio conocimiento acerca del tratamiento farmacológico y no farmacológico para no solo seguir las indicaciones médicas en el manejo del mismo, sino para aportar información e intervenir en el momento en que el tratamiento no esté dando resultados. Para hacer una correcta intervención farmacológica, el profesional ha de estar constantemente actualizado acerca de las últimas guías en el manejo del mismo, conocer las características de cada fármaco, no solo para poder ser un agente activo en el manejo, sino para educar al paciente sobre lo que debe esperar del fármaco y que este al mismo tiempo se convierta en un referente para decidir si continuar o no con el tratamiento. También es importante el conocimiento de tratamiento no farmacológico, ya que es un complemento que logra mejorar el bienestar del paciente (52).

- Conocer el sistema de salud y las políticas que lo rigen en cuanto a los tratamientos farmacológicos: Un escaso conocimiento de las regulaciones sobre sustancias controladas, y de regulación restrictiva de las sustancias controladas pueden convertirse en un obstáculo para manejar el dolor de una manera eficiente (36).

\section{Conclusiones}

La revisión permite concluir que el rol del profesional de enfermería en el manejo del dolor oncológico es deficiente, puesto que el nivel de conocimientos evidenciado en la literatura localizada es insuficiente, en aspectos que van desde el desconocimiento de las medidas no farmacológicas, hasta efectos adversos de los medicamentos, incluyendo las creencias que el paciente tiene sobre el dolor, entre otros. Por consiguiente, todos estos factores que influyen negativamente en el manejo del dolor oncológico, hacen que el paciente siga experimentando el dolor en diversos grados, donde el $38 \%$ de los pacientes oncológicos en todos los estadios experimentan dolor y el $80 \%$ de ellos en fases avanzadas, sufren un dolor en grado moderado a intenso según la OMS (46).

El manejo del dolor es un reto diario para el profesional de enfermería, especialmente cuando el cáncer es el causante del mismo. El cáncer es una de las principales causas de muerte en el mundo, siendo el dolor, el síntoma más frecuente en estos pacientes. Desde esta perspectiva el personal de enfermería cumple un rol importante en el cuidado y manejo del dolor. Por eso, es imprescindible que el profesional de enfermería tenga conocimientos amplios, actitudes y destrezas al momento de hacer una correcta valoración del paciente con dolor, además manejar el mimo con las mejores evidencias que le permitan mejorar su práctica profesional evidencia saber qué recursos usar, cuáles son las intervenciones adecuadas, hacer una revaloración después de las intervenciones y seguimiento del dolor de forma individual.

Se requiere que el profesional de enfermería posea conocimientos sobre el manejo del dolor oncológico, ya que es parte del equipo médico y es quien asiste permanentemente al paciente, lo que le permite determinar la eficacia del tratamiento y comentar la evolución para hacer los respectivos cambios. El escaso conocimiento, del profesional de enfermería incide en la disminución de la calidad de vida de los pacientes hace que su sufrimiento se prolongue $\mathrm{y}$, si el dolor persiste, puede deteriorar con el tiempo el estado físico y psicológico del paciente e incluso conducir a errores que pueden causar su muerte.

No se debe olvidar que la calidad de vida de los pacientes es uno de los objetivos primordiales de los tratamientos, ya que los pacientes cancerosos con dolor se deprimen, se vuelven ansiosos y cada vez más emotivos, pero si se logra aliviar de modo efectivo el dolor, todas estas alteraciones psicológicas revierten casi totalmente. La empatía y el apoyo se debe incluir igualmente sin olvidar el objetivo central de la medicina, que de acuerdo a Hipócrates dice "Curar, cuando 
no se puede aliviar", y siempre consolar y acompañar" (10), lo cual debe motivar al profesional de enfermería a estar en constante actualización para que de esta manera esté preparado para asumir los retos que cada día conlleva el manejo del dolor de un paciente oncológico.

Las enfermeras pueden hacer la diferencia entre un paciente que sufre hasta el último momento de su vida y un paciente que se siente cómodo y muere sin dolor y con dignidad. Sí, nosotros, los enfermeros podemos hacer la diferencia y tenemos que hacerla.

\section{Conflicto de intereses}

Los autores declaran que no tienen ningún conflicto de intereses en la realización de este artículo.

\section{Referencias}

1. Organización Mundial de la Salud. Nota descriptiva $\mathrm{N}^{\circ}$ 297. Estados Unidos de América: Organización Mundial de la Salud; 2015. Disponible en: http://www.who.int/ mediacentre/factsheets/fs297/es/.

2. Centro para el Control y la Prevención de Enfermedades. Estadísticas globales sobre el cáncer. Estados Unidos de América: Centro para el Control y la Prevención de Enfermedades; 2015. Disponible en: http://www.cdc.gov/spanish/cancer/international/statisti cs.htm.

3. Cajamarca-Barón JH. El cáncer y su impacto en salud pública. MedUNAB 2014; 17(1): 41-45.

4. International Agency for Research on Cancer. All Cancers (excluding non-melanoma skin cancer). Estimated Incidence, Mortality and Prevalence Worldwide in 2012. United States of America: International Agency for Research on Cancer; 2016. [Acceso 12 de febrero 2016]. Disponible en: http://globocan.iarc.fr/Pages/ fact sheets cancer.aspx

5. Ministerio de Salud y Protección Social. Instituto Nacional de Cancerología. Plan nacional para el control de cáncer en Colombia 2012-2020. Año 2012:11p. [Acceso 12 febrero 2016]. Disponible en: http://www. minsalud.gov.co/Documentos\%20y\%20Publicaciones/ Plan\%20nacional\%20para\%20el\%20control\%20del\%2 0c\%C3\%A1ncer\%20en\%20Colombia.pdf

6. Osma Zambrano SE, Uribe C. Tasas de incidencia y mortalidad por Cáncer de Mama en el Área Metropolitana de Bucaramanga en el período 20012005. Medunab 2014; 16(3): 101-111.

7. Ministerio de Salud del Perú. Análisis de la situación del cáncer en el Perú 2013. [Acceso 3 agosto 2016]. Disponible en: http://www.dge.gob.pe/portal/docs/ asis_cancer.pdf

8. Twycross R, Wilcock A, Start-Toller Claire. Symptom Management in Advanced Cancer. $4^{a}$ ed.- 4 nd ed. United Kingdom. 2009.

9. Benítez-del-Rosario MA, Pérez-Suárez MC, Fernández-Días R, Cabrejas-Sánchez A. Diagnóstico y tratamiento del dolor oncológico crónico (I). Aten Primaria 2002; 29(6): 374-377.
10. Astudillo W, Mendinueta C, Astudillo E, Gabilondo S. Principios básicos para el control del dolor total. Rev Soc Esp Dolor 1998;(6): 29-40.

11. Castillo-Garcet MA, JiménezHernándezAI, Torres-Orue I. Atención integral de enfermería al paciente oncológico en estadío terminal (2005). Rev Cubana Enfermer 2007; 23(2). Disponible en: http://www.bvs.sld.cu/revistas/enf/ vol23 2 07/enf02207.htm

12. Cruz-Hēnández JJ, González-Sarmiento R, LlorcaRamón G, Casinello-Espinosa J, Campos-Herrero C. Dolor oncológico. Reunión de expertos. Cátedra Extraordinaria del Dolor Fundación Grunenthal Universidad de Salamanca; 2005; Salamanca, España. Disponible en: http://www.fundaciongrunenthal.es/cms/ cda/file/Dolor+Oncol\%C3\%B3gico+.pdf?filelD=581001 95\&cacheFix=1238142767000\&_k=d48a4390180895 $5 a e 175 e b 0013 e 8 d a 5 c$

13. Montealegre-Gómez DC. Retos para enfermería en el cuidado de personas con dolor: una forma de humanización. Rev Cuid 2014; 5(1):679-88.

14. López L. La competencia cultural, una clave para mejores resultados en salud. Medunab 2016; 18(3): 263165.

15. Puebla-Díaz F. Tipos de dolor y escala terapéutica de la OMS. Dolor iatrogénico. Oncología 2005; 28(3): 33-37.

16. Sociedad Española de Oncología Médica. El dolor en el paciente oncológico. [Acceso 13 febrero 2016]. Disponible en: http://www.seom.org/seomcms/images/ stories/recursos/infopublico/publicaciones/F_Oncovida 17_BAJA.pdf

17. Macintyre PE, Walker S, Power I, Schug SA. Editorial: Acute Pain Management: Scientic Evidence revisited. BJA2006; 96(1): 1-4.

18. Palanca-Sánchez I, Puig-Riera de Conías MM, ElolaSomoza J, Bernal-Sobrino JL, Paniagua-Caparrós JL, Grupo de Expertos. Unidad de tratamiento de dolor: estándares y recomendaciones. Madrid: Ministerio de Sanidad, Política Social e Igualdad; 2011.

19. Merskey H. Classification of chronic pain. Descriptions of chronic syndromes and definitions. Pains 1986; S3: 5345-5356.

20. International Association for the Study of Pain. Caracterización neurobiológica del dolor visceral. 2012. [Acceso 03 de febrero 2016]. Disponible en: http://portal.sedolor.es/contenidos/273/adjuntos/fs03rk m6.pdf

21. Zegarra-Pierola JW. Bases fisiopatológicas del dolor. Acta Méd. Per 2007; 24(2):105-108.

22. Quijano AL, Montes MJ, Retamoso I. Tratamiento del dolor neuropático. Arch Med Int 2010; 32(1): 17-21.

23. Bader P, Echtle D, Fonteyne V, Livadas K, Meerleer G, Paez-Borda A, et al. Guía clínica sobre el tratamiento del dolor. European Association of Urology 2010. 2010; p. 1185 - 271. Disponible en: http://uroweb.org/wpcontent/uploads/16-GUIA-CLINICA-TRATAMIENTODOLOR1.pdf

24. Pernia A, Torres LM. Tratamiento del dolor oncológico intenso. Disponible en: http://www.paliativossinfronteras. com/upload/publica/dolor/programas-nacionales/ tratamiento_del_dolor_oncologicos_intenso.pdf

25. Achury-Saldaña DM. Dolor: la verdadera realidad. Aquichan 2008; 8(2):146-158.

26. Díaz M, Gattas S, Lopez JC, Tapica A. Enfermería oncológica: estándares de seguridad en el manejo del 
paciente oncológico. Revista Médica Clínica Las Condes 2013:24(4): 694-704.

27. Cancer Care Ontario. Institute for Clinical System Improvement. 2008

28. Espinoza-Venegas M, Valenzuela-Suazo S. Análisis de la Teoría de los Síntomas Desagradables en el Cuidado de la Enfermería Paliativa Oncológica. Rev Cubana Enfermer 2011; 27(2): 141-150.

29. NANDA Internacional. (2010). Diagnósticos enfermeros. Definiciones y clasificación. 2012-2014. Madrid: Elsevier.

30. Bulechek G, Butcher H, Dochterman J, Wagner C. Clasificación de intervenciones de enfermería (NIC). $6^{\text {a }}$ Edición. España: Elservier. 2014: 310-311.

31. Moorhead S, Johnson M, Mass-Meridean L, Swanson E. Clasificación de resultados de enfermería (NOC). $4^{a}$ Edición. España: Elservier. 2009. 303-305.

32. Albornos-Muñoz L, González-María E, Moreno-Casbas T. Implantación de guías de buenas prácticas en España. Programa de centros comprometidos con la excelencia en cuidados. Medunab 2015; 17(3):163-169

33. Grinspun D, Lloyd M, Xiao S, Bajnok I. Measuring the Quality of Evidence-Based Nursing Care: NQuIRE Nursing Quality Indicators for Reporting and Evaluation Data-System. Medunab 17(3):170-175

34. Rita W, Irmajean B, Tanya Ca. Promoting EvidenceBased Care through Nursing Order Sets. Medunab 17(3):176-181

35. Polit $D$, Hungler $B$. Investigación científica en ciencias de la salud. Principios y métodos. McGraw-Hill interamericana $6^{\circ}$ edición 2005:323-325.

36. Ruzafa-Martinez M, Lopez-Iborra L, Moreno-Casbas T, Madrigal-Torres M. Development and validation of the competence in evidence based practice questionnaire (EBP-COQ) among nursing students. BMC Medical Education 2013; 13: 19. e1-10.

37. Ho SE, Ho CC, Pang Yuen H, Lexshimi R, Choy YC, Jaafar MZ, Cardosa M, Das S. Clin Ter. A study of knowledge and attitudes of registered nurses towards pain management in an urban hospital. 2013 ;164(3):215-9. doi: 10.7417/CT.2013.1551.

38. Acuña L, Aibar S, Broggi L, Celano C, Cullen C, Chambi $\mathrm{MC}$, et al. Manual de enfermería oncológica. Buenos Aires: Instituto Nacional del Cáncer: 83p. Disponible en: http://www.msal.gob.ar/images/stories/bes/graficos/000 0000510cnt-38-ManualEnfermeriaOncologica2014.pdf

39. Uribe-Manrique E. Barreras del personal de salud que dificultan el control del dolor en el paciente oncológico. [Tesis]. Bogotá: Biblioteca General, Universidad Pontificia Javeriana; 2008.

40. Jensen MP, Karoly P. Self- report scales and procedures for assessing pain in adults. Handbook of pain assessment. Third Edition. Nueva York: Guilford; 2001: 19-35

41. Salvadó-Hernández C, Fuentelsaz-Gallego C, ArcayVeira C, López-Meléndez C, Villar-Arnal T. Conocimientos y actitudes sobre el manejo del dolor por parte de las enfermeras de unidades de cirugía y oncología de hospitales nivel III. Enferm Clin. 2009; 19(6):322 -29.

42. Bruera E, Willey JS, Ewert-Flannagan PA, Cline MK, Kaur G, Shen L, Zhang T, Palmer JL. Pain intensity assessment by bedside nurses and palliative care consultants: a retrospective study. Support Care Cancer 2005; 13(4): 228 - 231

43. Ministerio de Salud. Alivio del dolor por cáncer avanzado y cuidados paliativos. Santiago de Chile; 2011. Disponible en: http://web.minsal.cl/portal/url/ item/72213ed52c2723d1e04001011f011398.pdf

44. Zavala-Rodríguez MR, Fang-Huerta MA, Ortega-López RM, Rivera-Villegas B. Manejo del dolor por el personal de enfermería. Dol Clin Ter 2005; 4(1):11-16.

45. McCaffery M, Pasero C. Pain: Clinical Manual (2nd ed.). (1999).St. Louis: Mosby: 35-102.

46. Bernardi M, Catania G, LambertA, Tridello G, Luzzani M. Knowledge and attitudes about cancer pain management: A national survery of Italian oncology nurses. European Journal of Oncology Nursing. 2007; 11(3): 272-279.

47. González-Escalada JR, Camba A, Casas A, Gascón P, Herruzo I, Núñez-Olarte J, Ramos-Aguerri J, Trelis, Torres LM. Código de buena práctica para el control del dolor oncológico. Rev. Soc. Esp. Dolor 2011; 18(2): 98117.

48. Gonzales-Elías SE, Jorge-Martínez YC, Lovo-Granados JM, Rivera-Guardado BL. Conocimiento que poseen los profesionales de enfermería sobre las intervenciones para el alivio del dolor como parte de los cuidados paliativos, a pacientes con patologías en fase terminal en los servicios de medicina I, II y oncología del Hospital Médico Quirúrgico y Oncológico del Instituto Salvadoreño del Seguro Social. [Tesis licenciatura]. Salvador. Universidad de el Salvador; 2010.

49. Deng G, Cawhisth BR. Integrative oncology; complementary therapies for pain anxiety and mood disturbance. CA Cancer. J Clin 2005; 55:109-16.

50. Miró J. Evaluación de la intensidad del dolor en el paciente con cáncer. Psicooncología. 2012; 9(2-3): 393401.

51. Mahfudh SS. Nurse's role in controlling cancer pain. J Pediatr Hematol Oncol 2011; 33(2): S146-8.

52. García- Armenta E. Uso de combinaciones de medicamentos para el manejo del dolor y la inflamación. Rev Fac Med UNAM. 2000 43(5):209-211. 